\title{
Direct observation of lasing mode in a microdisk laser by a near-field-probing technique
}

\author{
Toshihiko Baba, ${ }^{\text {a) }}$ Hiroshi Yamada, and Atsushi Sakai \\ Yokohama National University, Division of Electrical and Computer Engineering, 79-5 Tokiwadai, \\ Hodogayaku, Yokohama 240-8501, Japan
}

(Received 23 May 2000; accepted for publication 12 July 2000)

\begin{abstract}
Evanescent field profiles of whispering gallery (WG) modes in a 1.6- $\mu \mathrm{m}-\mathrm{GaInAsP}$ microdisk injection laser were investigated by scanning a nanoprobe near the periphery of a lasing microdisk. The increase in threshold current due to the light scattering by the probe and the corresponding decrease in laser light were observed experimentally. Two-dimensional images of the evanescent field, which agreed well with theoretical expectations, were obtained by the use of a Pt-Ir probe. The images indicate that the WG mode is strongly locked by the fourfold symmetry in the microdisk with center post claddings. This characteristic is favorable for the microdisk laser itself to be used as an active near-field probe. A lower contrast image obtained by the use of a tapered silica fiber probe suggests that the microdisk probe can be sensitive to both surface profiles and optical properties of the object. (C) 2000 American Institute of Physics. [S0003-6951(00)01737-X]
\end{abstract}

Semiconductor microdisk lasers ${ }^{1-5}$ have whispering gallery (WG) modes as lasing modes. They are strongly confined in a disk active layer by the total internal reflection at semiconductor/air boundaries. This causes the difficulty in extracting efficient light output from this type of laser. On the other hand, this feature can be used for a sensing, in which a lasing microdisk acts as an active near-field optical probe. ${ }^{6}$ Since WG modes have the evanescent field spreading to the air, it suffers scattering loss when an object is put close to the periphery of the microdisk. Then, the surface profile of the object is converted to the modulation in threshold current and in laser light. This probe is advantageous due to (1) the multifunction of the microdisk laser, i.e., light emission, probing, and amplification, and (2) a micrometer-order small size suitable for large-scale integration and parallel probing.

In a previous study, we experimentally observed the response of the laser light against a metal needle moving one dimensionally against a microdisk in the direction normal to the disk plane. ${ }^{7}$ It was not precisely the probing of the shape of the needle tip by the microdisk probe, but rather the probing of the evanescent field of the WG mode by the metal probe. This is because the tip radius of the metal needle, 50 $\mathrm{nm}$, was much smaller than the full width at half maximum (FWHM) of the response, $160 \mathrm{~nm}, \sim 0.1$ times the lasing wavelength $1.55 \mu \mathrm{m}$. However, the result suggested the microdisk probe action well and also suggested the potential of the probing of mode fields in various lasers by this method. In this study, we developed the scanning system for twodimensional (2D) probing. This enabled us to investigate the behavior of lasing characteristics influenced by the probe and a detailed profile of the evanescent field. They exhibited some very unique properties, indicating the clear evidence of the operation principle of the microdisk probe and the strong lock of the mode standing wave in a microdisk laser with center post claddings.

Figure 1 shows the setup for the probing experiment and

${ }^{a)}$ Electronic mail: baba@dnj.ynu.ac.jp the coordinate system. We fabricated an injection-type microdisk laser with a GaInAsP/InP compressively strained multiquantum wells by inductively coupled plasma etching, as described in Ref. 4. The diameter and the thickness of the microdisk were 6 and $0.2 \mu \mathrm{m}$, respectively. The current was flowed from a Pt-Ir needle (commercially available for scanning electron microscopes) to the top contact layer (omitted in Fig. 1). The lasing wavelength was $1.58-1.60 \mu \mathrm{m}$, and the threshold current was typically $0.5 \mathrm{~mA}$ under pulsed condition at room temperature. The stable single mode operation was observed in all the experiments in this study. For the probing experiment, the laser was biased above threshold and the light radiated almost uniformly around the microdisk by the scattering at the imperfect disk edge was detected by a tapered multimode fiber. The detected light was spectrally resolved by an optical spectrum analyzer and the intensity at the lasing wavelength was recorded as the light intensity. For the probing of the evanescent field of the WG mode, another $\mathrm{Pt}-\mathrm{Ir}$ needle with a tip radius of $\sim 150 \mathrm{~nm}$ was first used as a probe. It was electrically connected to the ground level to minimize the electrostatic force. The position of the needle tip was controlled by three piezo stages each having $15 \mathrm{~nm}$ resolution. The reciprocating motion of the needle tip inside the $x y$ plane, as illustrated in Fig. 1, was programmed in a

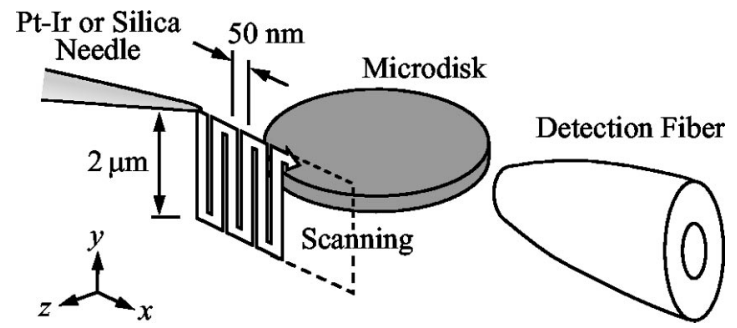

FIG. 1. Schematic of the measurement setup. Near the periphery of a microdisk injection laser, a sharp needle was scanned multidimensionally. The change of the laser light was detected by a tapered multimode fiber put close to the microdisk. All the measurements were carried out at room temperature by the pulsed injection current of $200 \mathrm{~ns}$ pulse width and $2 \mathrm{kHz}$ repetition frequency. 


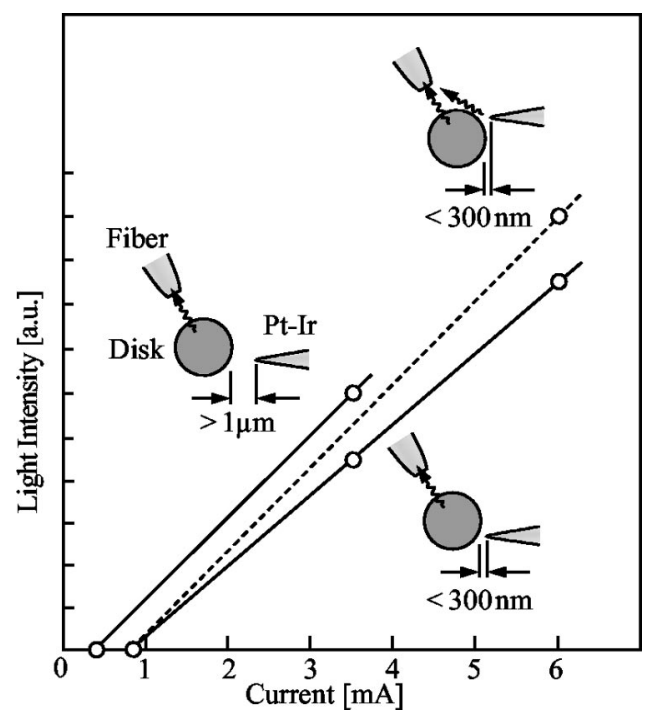

FIG. 2. Lasing characteristics observed against three different relative positions of the microdisk, the fiber, and the Pt-Ir needle having $\sim 150 \mathrm{~nm}$ tip radius.

computer for a 2D imaging of the evanescent field.

Figure 2 shows light versus current characteristics, which were influenced by positions of needle and fiber tips. The threshold current was increased to nearly twice at $z$ $\sim 300 \mathrm{~nm}$. This simply shows the operation principle of the microdisk probe. When the needle tip was close to the fiber tip, the light was increased even though the threshold current was not changed. This was caused by the direct insertion of the scattered light by the needle into the fiber. Figure 3 shows the light observed with various distances $z$ at relative position of the probe and the fiber, as shown in the lower inset of Fig. 2. The light drastically decreased when $z$ $<300 \mathrm{~nm}$. The length $z \sim 300 \mathrm{~nm}$ should be a decay length of the evanescent field.

Figure 4 shows responses of light when the Pt-Ir probe was moved two dimensionally inside the $x y$ plane. Even at $z \sim 500 \mathrm{~nm}$, which is much larger than the decay length of

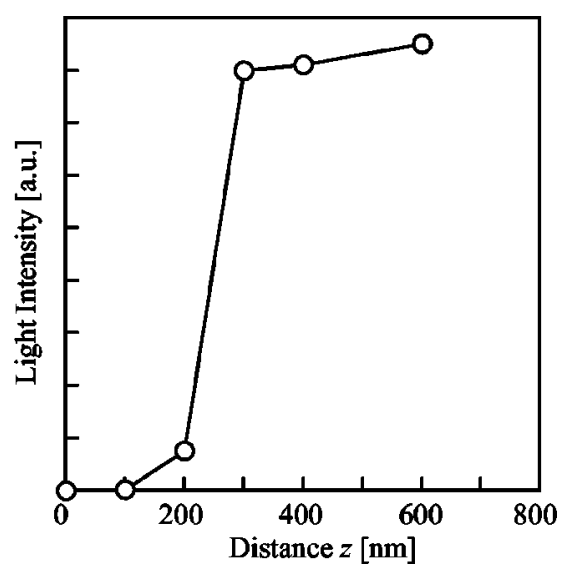

FIG. 3. The change of laser light with distance $z$ between the periphery of the microdisk and the Pt-Ir needle tip. The distance $z$ was measured from a point, at which the needle was touched to the microdisk. The estimation of the position included $<50 \mathrm{~nm}$ error, since the lasing characteristic did not change drastically even when the needle touched the microdisk. The touching was sensed from the gradual decrease in detected light. It was caused by the push of the microdisk by the needle and the change of the relative

the lasing always occurs by fourfold symmetric modes under
Downloaded 01 Feb 2007 to 133.34.171.229. Redistribution subject to AIP license or copyright, see http://apl.aip.org/apl/copyright.jsp

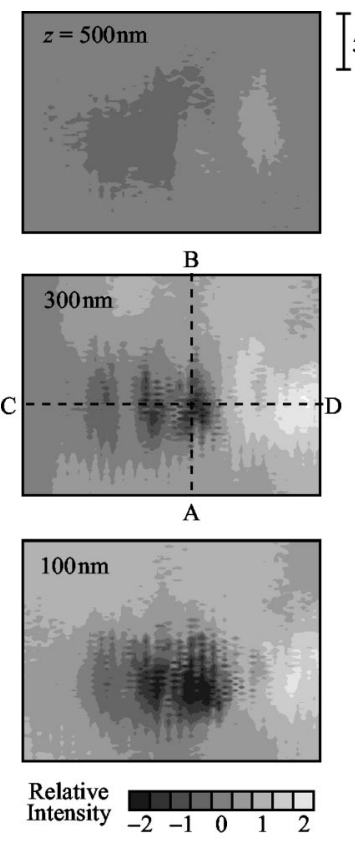

(a)

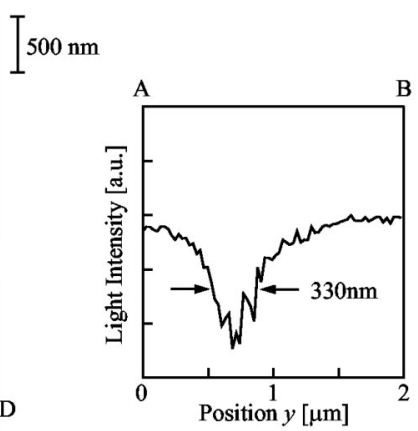

(b)

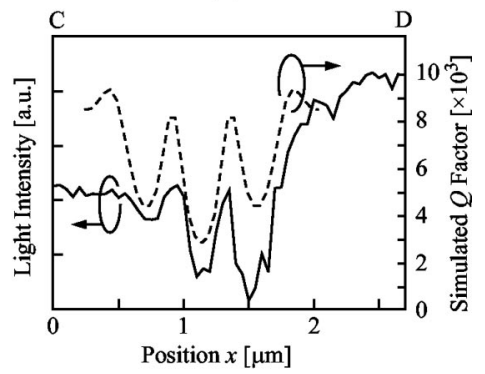

(c)
FIG. 4. Responses of light against the Pt-Ir needle having $\sim 150 \mathrm{~nm}$ tip radius. (a) shows those against the needle moving inside the $x y$ plane with various distances $z$. The zero relative intensity corresponds to the light lower two figures were caused by the reciprocating motion of the needle, as illustrated in Figs. 1(b) and 1(c) are responses along lines A-B and C-D in (a), respectively. The dashed line in (c) indicates the quality factor $Q$ of the microdisk neighbored with a metal needle, which is calculated by the twodimensional finite difference time domain method with the equivalent refractive index approximation.

the evanescent field, the influence of the probe is clear, as shown in Fig. 4(a). At $z \sim 300 \mathrm{~nm}$, a minimum of light intensity was observed in the direction normal to the disk plane $(y$ direction) with FWHM of $330 \mathrm{~nm}$. This FWHM is much wider than $160 \mathrm{~nm}$ obtained against a needle with a tip radius of $50 \mathrm{~nm}^{7}$ Therefore, it should correspond to the size of the probe tip, not the distribution of a WG mode. In Fig. 4(c), one maximum and three minima are observed along the line parallel to the disk plane ( $x$ direction). As explained above, the maximum at the right end of this figure is due to the increase in direct light scattered by the probe and coupled into the fiber. On the other hand, it is reasonable to consider that three minima were due to the standing wave of a WG mode. However, a more complex discussion is needed to explain this result. Suppose that there are no intrinsic mechanisms that lock positions of nodes and antinodes of the standing wave in a microdisk laser with perfect axial symmetry. Then, the positions should be changed by the moving needle so that the scattering loss of the WG mode is minimized. This would extinguish the response displaying the standing wave. However, such a response was actually observed at $z \sim 300 \mathrm{~nm}$. Thus, we should conclude that the standing wave is intrinsically locked in an actual microdisk laser. This conclusion is verified by a theoretical calculation; the observed response agrees well with the calculated one by the finite difference time domain (FDTD) method, as shown in Fig. 4(c), assuming that the position of the standing wave is locked. It is consistent with the discussion in Ref. 5, i.e., the lasing always occurs by fourfold symmetric modes under AIP license or copyright, see http://apl.aip.org/apl/copyright.jsp intensity without the influence of the needle. Vertical stripe patterns seen in 


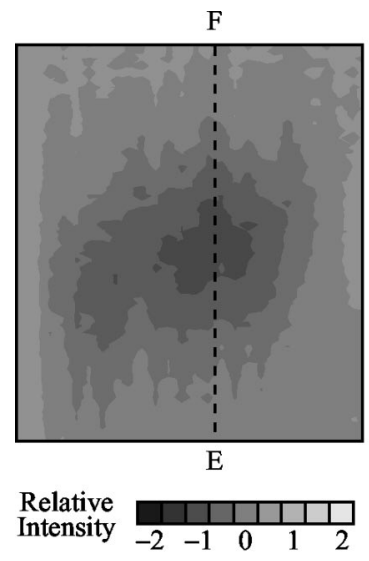

(a)

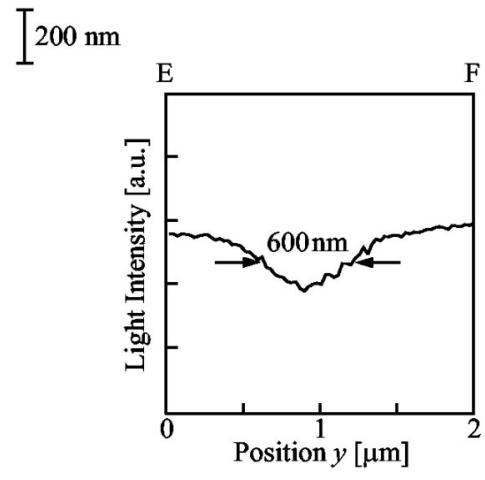

(b)
FIG. 5. Response of light against a tapered silica fiber having $\sim 400 \mathrm{~nm}$ tip radius at $z \sim 300 \mathrm{~nm}$ and the change of the light intensity along line $\mathrm{E}-\mathrm{F}$.

the influence of the center square post claddings supporting the microdisk. As seen in Fig. 4(a), the response still displays the standing wave at $z \sim 100 \mathrm{~nm}$. The lock of the standing wave is much stronger than the influence of the moving needle. This characteristic is favorable not only for the nearfield probe action but also for the reduction in threshold current, since the unwanted stimulated emission for degenerate nonlasing modes $^{8}$ is suppressed by the lock of the standing wave.

Figure 5 shows responses against a tapered silica fiber used in place of the needle. Since the tip radius of the fiber was $\sim 400 \mathrm{~nm}$, much larger than the period of the standing wave, the 2D response in Fig. 5(a) displays the profile of the fiber tip. For the fiber, the contrast of the minimum to the background level was $1 / 3$ of that for the Pt-Ir needle. This is qualitatively consistent with a result of the FDTD simulation, i.e., for $z>200 \mathrm{~nm}$ (weak scattering regime), a lower dielectric constant of the object causes a smaller scattering loss of the WG mode and hence a lower image contrast. The simulation also suggests that a distance $z$ less than $100 \mathrm{~nm}$ rather changes the profile of the WG mode and causes a coupling of the WG mode to the object, which make the relation more complex.
In conclusion, we demonstrated the direct observation of the evanescent field profiles of the WG mode in a microdisk injection laser by a near-field probing technique. Obtained profiles agreed well with theoretical expectations. They indicated that the position of the mode standing wave was strongly locked by the square post claddings supporting the microdisk. The circular image corresponding well to the shape of the tapered silica fiber with $\sim 400 \mathrm{~nm}$ tip radius clearly demonstrated the active near-field probe action in the microdisk laser. The lower image contrast against the fiber compared with that against the metal, which was observed in the weak scattering regime, suggested the potential of a multifunctional probe sensitive to both surface profiles and optical properties.

The authors would like to thank Professor Y. Kokubun, Yokohama National University, and Professor K. Iga, Professor S. Arai, Professor F. Koyama, and Associate Professor T. Miyamoto, Tokyo Institute of Technology, for encouragement and helpful suggestions. They also thank Dr. A. Kasukawa, The Furukawa Electric Co., Ltd., for help with the experiment. This work was partly supported by The Grantin-Aid No. 10450025 by the Ministry of Education, Science, Sports and Culture, and also supported by Japan Society for Promotion of Science, Research for the Future No. JSPSRFTF 97P00103.

\footnotetext{
${ }^{1}$ A. F. J. Levi, R. E. Slusher, S. L. McCall, T. Tanbun-Ek, D. L. Coblentz, and S. J. Pearton, Electron. Lett. 28, 1010 (1992).

${ }^{2}$ T. Baba, M. Fujita, A. Sakai, M. Kihara, and R. Watanabe, IEEE Photonics Technol. Lett. 9, 878 (1997).

${ }^{3}$ M. Fujita, A. Sakai, and T. Baba, IEEE J. Sel. Top Quantum Electron 5, 673 (1999).

${ }^{4}$ M. Fujita, R. Ushigome, and T. Baba, Electron. Lett. 36, 790 (2000).

${ }^{5}$ M. Fujita, R. Ushigome, and T. Baba, IEEE Photonics Technol. Lett. (submitted).

${ }^{6}$ A. Sakai, H. Yamada, M. Fujita, and T. Baba, Jpn. J. Appl. Phys., Part 1 37A, 517 (1998).

${ }^{7}$ H. Yamada, A. Sakai, M. Fujita, and T. Baba, Electron. Lett. 27, 222 (1999).

${ }^{8}$ S. A. Backes, J. R. A. Cleaver, A. P. Heberle, J. J. Baumberg, and K. Köhler, Appl. Phys. Lett. 74, 176 (1999).
} 